\title{
Simultaneous Sensing of Dual Analyte with Photonic Crystal Fiber Based Liquid Sensor
}

\author{
H. ADEMGIL
}

\begin{abstract}
This article proposes three novel models of photonic crystal fiber (PCF) based dual liquid sensor. The performances of Z-Model, X-Model, and V-Model structures have been evaluated by applying the well-known full vectorial finite element method (FV-FEM) where boundaries are defined with perfectly matched layers (PMLs). The proposed models have been investigated with two types of analyte arrangements (A-Type and B-Type) in the core region. The birefringence and the sensitivity levels of proposed sensors have been studied where core holes are filled with water $(n=1.33)$ and ethanol $(n=1.354)$. Our numerical results have shown that X-Model and Z-Model of A-Type sensor is an ideal structure for water and ethanol sensing, respectively.
\end{abstract}

Index Terms - Birefringence, evanescent sensing, Optical Sensor, photonic crystal fiber

\section{INTRODUCTION}

$\mathrm{O}$ PTICAL FIBERS were initially established for telecommunication applications. However, their application areas are not limited with this sector. Progressively it can be employed in different areas competently. Photonic crystal fiber (PCF) is another class of optical fiber in light of the properties of photonic crystals [1]. They comprise of a silica core, encompassed by an occasional cluster of air-holes positioned alongside the full extent of the fiber structure. On account of its capacity to confine the light in hollow cores or with repression qualities unrealistic in traditional optical fiber, $\mathrm{PCF}$ is presently discovering applications in various sectors. These microstructured fibers have demonstrated virtues in various fields such as communication [1], nonlinear optics [2], high power technology [3] and sensing [4-5].

Features such as small dimension, light weight, electromagnetic interference resistance, potential for remote sensing and design flexibilities make PCFs stand out for sensing applications [6]. In PCFs, by modifying the structural design parameters such as hole to hole distances, air hole dimensions, air hole shape, and pattern geometry unique

HUSEYIN ADEMGIL, is with Department of Computer Engineering European University of Lefke, Lefke, Northeren Cyprus, TR-10 Mersin Turkey, (e-mail: hademgil@eul.edu.tr).

(iD) https://orcid.org/0000-0002-2520-8567

Manuscript received August 21, 2019; accepted September 25, 2019. DOI: $10.17694 /$ bajece. 608334 propagation features can be achieved. Previously conducted studies have demonstrated that by choosing the suitable design parameters it is possible to control some key propagation properties such as dispersion, birefringence, nonlinearity, and confinement losses for wide wavelength range [5, 7-8].

Previous studies have shown that liquid and gas sensors are the most desirable sensing samples in industrial and biological practices $[4,6]$. In recent years, especially PCF based liquid sensors have attracted great interest especially in bio/chemical solutions. Generally, water, alcohol or benzene types of materials are the major solutions utilized in these processes [9].

Hollow core Photonic Band Gap (PBG) and index guiding are the two well-known guiding mechanisms in PCF structures. It is well known that PBG type PCFs are an ideal candidate for gas sensing applications. Contrariwise, index guided PCF structures are appropriate for liquid sensing solutions. In PCF based sensors evanescent field is the vital component for sensing performance [10-11].

The optical mode of the index guided PCF structures are mainly confined at the centre of the core region. In index guided PCF based sensor designs, biological and chemical liquid samples (analyte) are hosted by air holes around the core region. In this regard, the sensing process is determined by the evanescent interaction between the guided mode and the analyte. Due to direct interaction of PCF core and sensing material (analyte) the evanescent PCF structures are considered as intrinsic sensors [10-11].

Initially, Monro et al. [11] proposed the evanescent-wave PCF sensor formation. Furthermore, Cordeiro et al. [10] has verified that the PCF structure with analyte filled holes are boosting the light mode interaction with the analyte to be detected.

Recent studies have demonstrated that different core and cladding configurations of PCF based sensors can be used for various sensing applications. Naeem et al. [12] have experimentally shown that two core PCF can be employed for strain and temperature sensing. Moreover, temperature and stress sensors with alcohol filled holes are experimentally demonstrated by Shi et al. [13] In addition, PCF structures for water-ethanol [14] and water, ethanol and benzyne [15] solutions are proposed theoretically. On the other hand, PCF structures in the terahertz spectrum are proposed theoretically for sensing blood components [16] and analytes such as ethanol and benzyne. Birefringence feature is commonly intended for polarization control in fiber based optical sensors and devices [17]. Additionally, highly birefringent fibers are emerging the polarization mode interference [18-19]. Such 
fiber structures are ideal candidate for hydrostatic pressure sensors [18]. The numerical results in Islam et al. [19] have shown that high birefringent chemical sensor with relatively high sensitivities can be achieved with rectangular structured air holes. However, on the experimental point of view, due to sharp edges of rectangular air holes and complex design parameters this structure can be challenging [19].

The intention of this work is to propose highly birefringent PCF based sensor with dual analyte sensing ability. Unlike previous studies, in order to achieve birefringence, the symmetry of the PCF structure is broken with different analyte arrangements in the core region. Best to my knowledge, dual analyte sensing with such PCF core arrangements have not been studied and compared in the past.

\section{DESIGN AND METHODOLOGY}

Fig. 1 expresses the geometry of the proposed design of PCF based optical sensors. In this work, numerical simulations are engaged with commercial software Comsol Multiphysics. FV-FEM is engaged to analyze the various propagation characteristics of PCF based optical sensor [7, 11]. Perfectly match layers (PML) are engaged as boundary condition. The composition of the cladding air holes is hexagonal, where silica $(\mathrm{n}=1.45)$ is used as background material. The cladding zone is formed by 3 rings of 54 circular air hole, where the hole diameter (d) is fixed to $2 \mu \mathrm{m}$, and hole to hole distance $(\Lambda)$ of cladding air holes is set to $2.4 \mu \mathrm{m}$. Proposed PCF structure contains 19 analyte filled holes in the core region where center to center distance of core holes is set to $1.4 \mu \mathrm{m}$. As can be seen from the fig. 1, internal core holes are filled with two different analytes. Structures are denoted as $Z$ Model, X-Model, and V-Model where core hole diameters are fixed to $1.2 \mu \mathrm{m}$. The simultaneous detection of two liquid analytes is possible with this core arrangement. Due to the asymmetric refractive index (RI) arrangement in the core region, considered amount of birefringence can also be achieved. The numerical results are calculated for two types of analyte arrangement (A-Type and B-Type). Analyte fillings are detailed in Table 1, where, Water $\left(\mathrm{n}_{\mathrm{a}}=1.33\right)$ and Ethanol $\left(\mathrm{n}_{\mathrm{a}}=1.354\right)$ are considered.

The structural geometry of PCF is very precise as the cross sectional area is divided into sub-domains. Numerical simulations of proposed PCF models are carried out with triangular sub-domains. The FV-FEM procedure is applied with Maxwell's equations. The well-known wave equation is written as $[7,10-11,20]$;

$$
\nabla \times\left([\mathrm{s}]^{-1} \nabla \times E-k_{0}^{2} n^{2}[s] E=0\right.
$$

the electric field vector is denoted by E, where the wave number in free space is $k_{0}=2 \pi / \lambda$ and $n$ is the refractive index of the silica background. The anisotropic PML is described with [s] and $\lambda$ is the operating wavelength.

Birefringence is one of the key parameters that is beneficial and advantageous for various applications of optical fibers. Birefringence is defined as the absolute difference of effective refractive index of $x$ and $y$ polarized fundamental modes [1719]:

$$
B=\left|n_{e f f}^{x}-n_{e f f}^{y}\right|
$$

$n_{e f f}^{x}$, and $n_{e f f}^{y}$ are denoting the effective refractive indices of polarization modes.

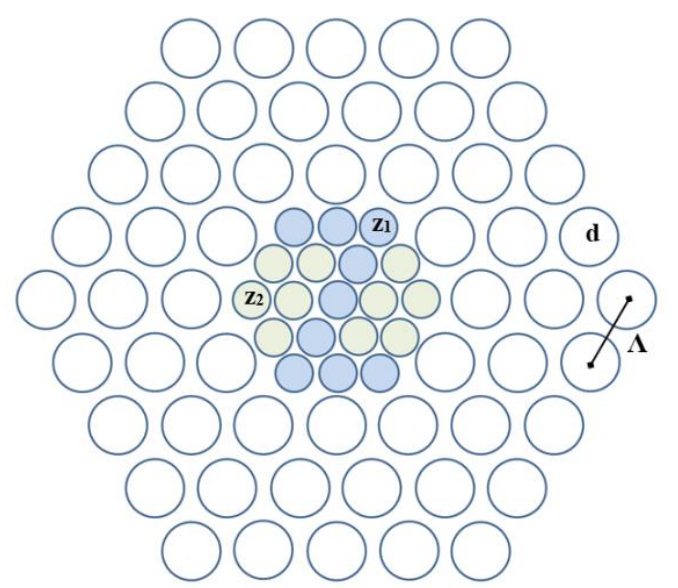

(a)
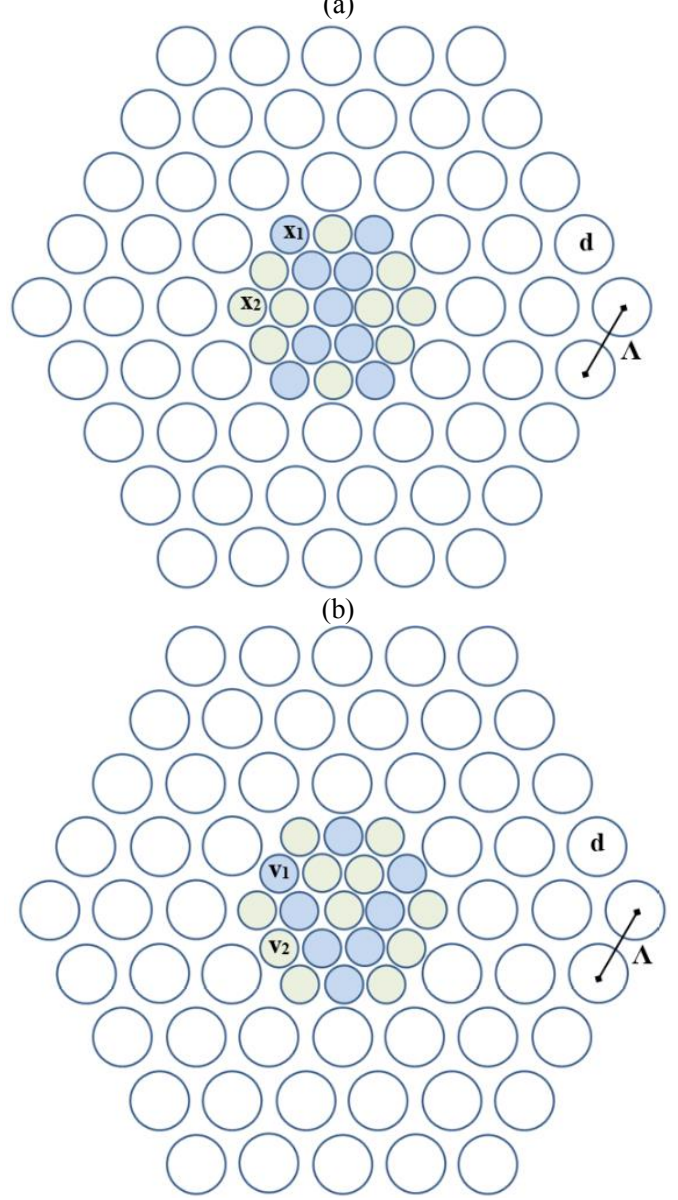

(c)

Fig.1. Schematic view of; (a) Z-Model PCF sensor, (b) X-Model PCF sensor and (c) $V$ - Model PCF sensor.

On the other hand, the sensing performance of the liquid sensor can be predictable by calculating the relative sensitivity of the introduced PCF models. Briefly, the interaction volume of the guided mode and liquid analyte is indicating the relative 
sensitivity. This key parameter can be mathematically described with the formula below [7, 10,19];

$r=\frac{n_{a}}{n_{e f f}} \times f$

The analyte index and the effective refractive index of the guided light mode are denoted with $n_{a}$ and $n_{\text {eff }}$, respectively. The ratio of the power flow through the specified domain is denoted as $f$, where it is calculated with the formula given below $[7,10,19]$;

$f=\frac{(\text { sample }) \int \operatorname{Re}\left(E_{x} H_{y}^{*}-E_{y} H_{x}^{*}\right) d x d y}{(\text { total }) \int \operatorname{Re}\left(E_{x} H_{y}^{*}-E_{y} H_{x}^{*}\right) d x d y} \times 100$

\section{NUMERICAL RESULTS AND DISCUSSION}

The dual analyte PCF models with analyte parameters presented in Table I are numerically investigated. It can be seen from the table that, in A-Type sensor models the primary core holes $\left(X_{1}, Z_{l}\right.$, and $\left.V_{l}\right)$ and secondary core holes $\left(X_{2}, Z_{2}\right.$, and $V_{l}$ ) are filled with 1.33 and $1.354 \mathrm{RI}$ analyte, respectively. On the other hand, analyte filling of core holes are contrary in B- Type sensor models.

TABLE I

PCF SENSOR ANALYTE ARRANGEMENTS

\begin{tabular}{|c|c|c|c|}
\hline \multicolumn{2}{|c|}{ Sensor Model } & $\begin{array}{c}\text { A-Type } \\
\text { Sensor }\end{array}$ & $\begin{array}{c}\text { B - Type } \\
\text { Sensor }\end{array}$ \\
\hline \multirow{2}{*}{$Z-$ Model } & $\mathrm{Z}_{1}$ & 1.33 & 1.354 \\
\cline { 2 - 4 } & $\mathrm{Z}_{2}$ & 1.354 & 1.33 \\
\hline \multirow{3}{*}{- Model } & $\mathrm{X}_{1}$ & 1.33 & 1.354 \\
\cline { 2 - 4 } & $\mathrm{X}_{2}$ & 1.354 & 1.33 \\
\hline \multirow{2}{*}{-Model } & $\mathrm{V}_{1}$ & 1.33 & 1.354 \\
\cline { 2 - 4 } & $\mathrm{V}_{2}$ & 1.354 & 1.33 \\
\hline
\end{tabular}

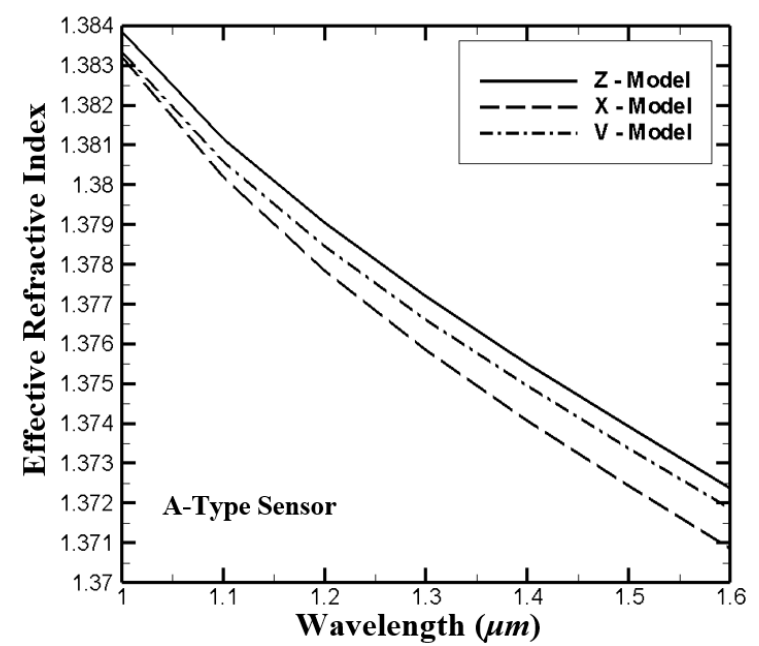

(a)

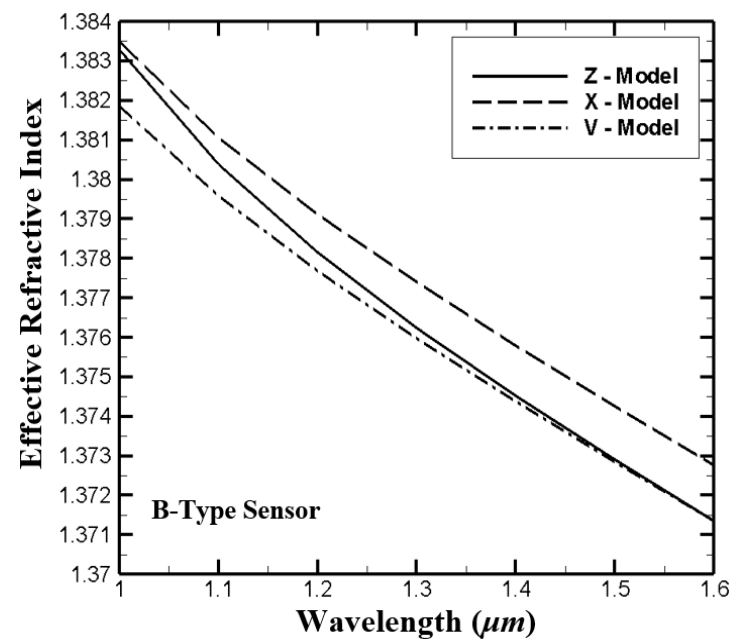

(b)

Fig. 2. Variations of effective refractive index for proposed $Z, X$ and $V$ models of PCF sensor.

The variations of effective refractive index of proposed structures relate with operating wavelength is presented in Fig. 2. These figures indicate that the effective refractive index of both types of sensors is decreasing with increasing wavelength. In $A$ - Type sensor, effective index of Z-Model is higher than other models, where $X$-Model reaches higher levels in B-Type sensor. It is worth noting that the effective refractive index of both sensor types are varying between 1.384- 1.371 for wide wavelength range.

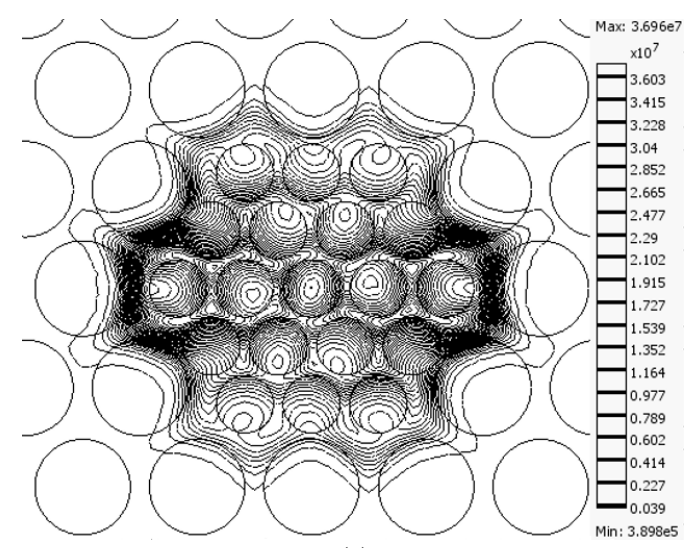

(a)

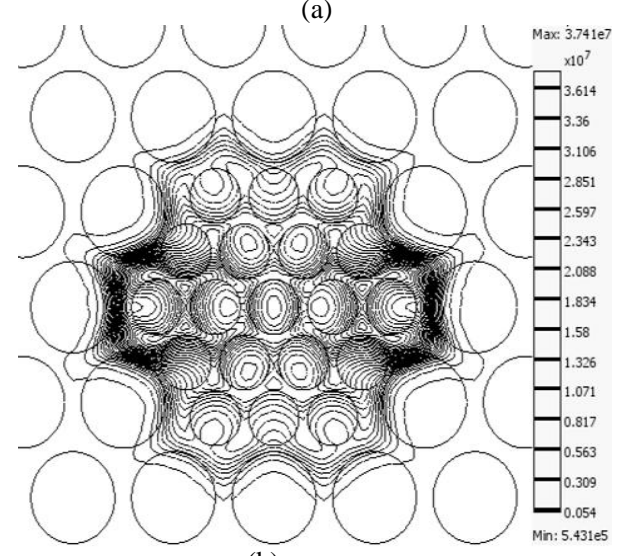

(b)

Fig. 3. Electric field distribution at $\lambda=1.1 \mu \mathrm{m}$, where, (a) $Z$ - Model and (b) $X$ Model of A-Type PCF sensor. 
The amount of light and liquid analyte interactions are presented in Fig. 3. Further, substantial interaction between the guided mode and analyte filled holes are illustrated. Figures show the field profile of the $x$-polarized $\mathrm{HE}_{11}$ mode for Z-Model and X-Model of A-Type PCF sensor. The amount of light mode and analyte interaction can be associated with the level of sensitivity coefficient of the PCF sensor models.

The birefringence is the momentous propagation feature of the PCF based optical sensors. The asymmetric liquid fillings of core holes are triggering refractive index differences between the $x$-y polarized modes. As expected, considered amount of birefringence can be realised in Fig. 4 (a) \& (b). The V-Model sensor contains the lowest birefringence values for both sensor types. On the other hand, X- Model and Z- Model show similar performances for both types. However, it is worth noting that birefringence level of proposed structures reaches 2 times improved performance with A- Type sensor. Fig.4 (b) indicates that, at shorter wavelengths birefringence of $\mathrm{Z}$ Model is slightly higher than X-Model where this is contrary at longer wavelengths. This variation can be associated to the effective refractive index differences of $x-y$ polarized modes where $x$ - polarized mode of Z-Model is getting closer to $y$ polarized mode at longer wavelengths.

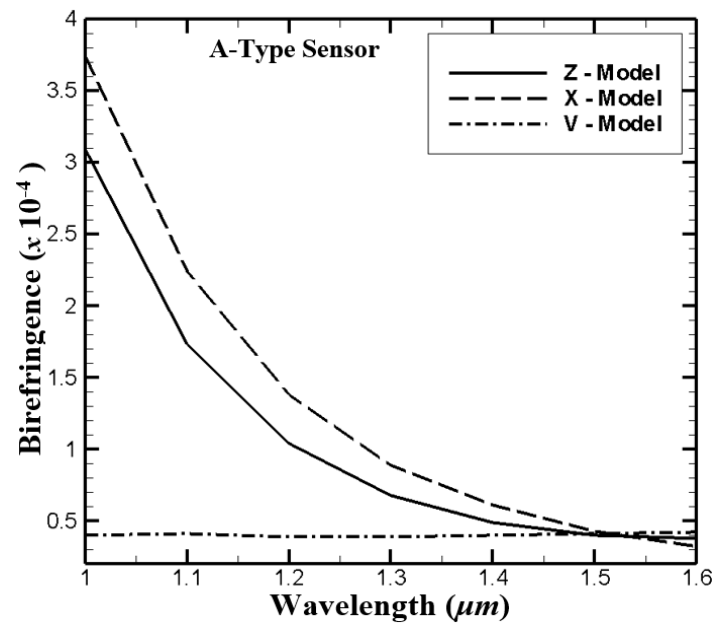

(a)

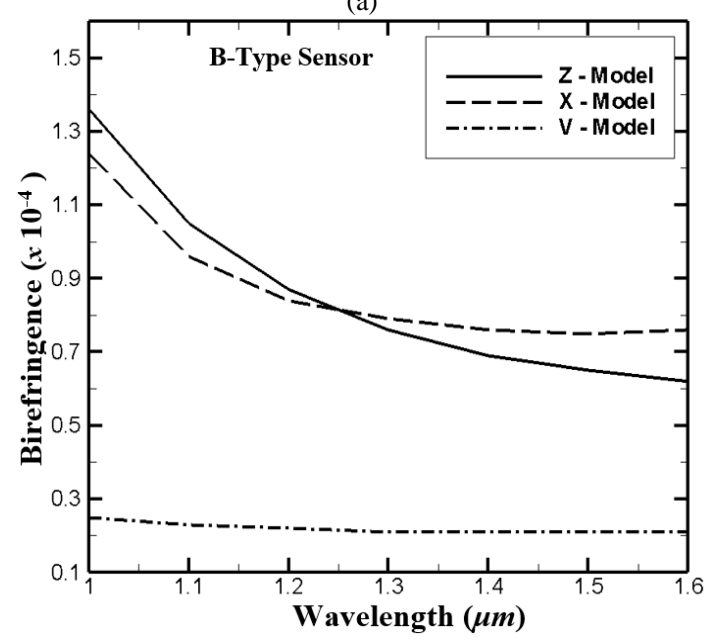

(b)

Fig. 4. Variations of birefringence for proposed $Z, X$ and $V$ models of PCF sensor.
Finally, Fig. 5 (a) \& (b) shows the variations of sensitivity coefficient with respect to operating wavelength. It can be observed from both figures that the relative sensitivity is increasing at longer wavelengths. This phenomenon can be linked to improved interaction between analyte and guided mode. As expected $[7,10,19]$, sensitivity coefficient level of higher index analyte (Ethanol) is moderately higher than lower index analyte (Water). Our proposed structures are almost 2 times more sensitive to Ethanol compared to water at broad wavelength range.

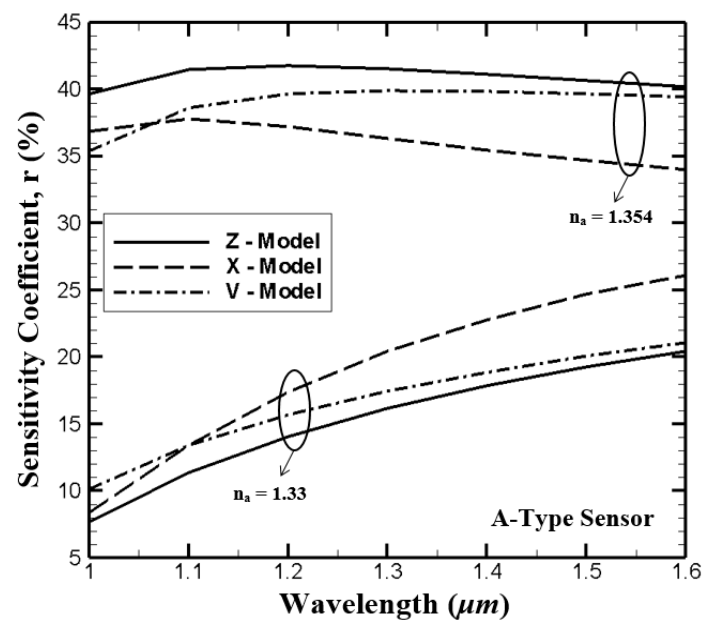

(a)

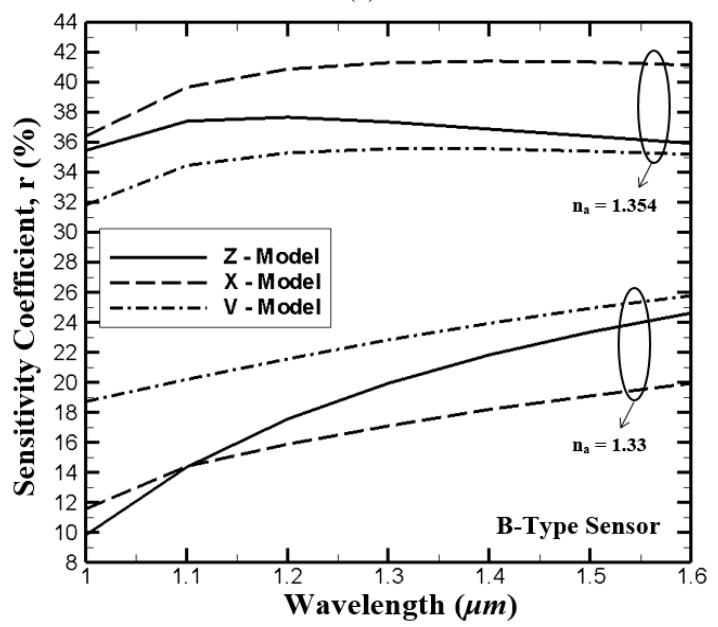

(b)

Fig. 5. Variations of sensitivity coefficients for proposed $Z, X$ and $V$ models of PCF sensor.

Table II briefly describes the overall performance of sensor types. It can be seen that X-Model of A-Type sensor shows overall better performance (high birefringence and high sesnsitivity) for water $\left(\mathrm{n}_{\mathrm{a}}=1.33\right)$ sensing. On the other hand, Z-Model of A-Type sensor is more desirable for ethanol $\left(\mathrm{n}_{\mathrm{a}}=\right.$ 1.354) sensing. More specifically, if we are only considering the sensitivity, Z-Model of A-Type and X-Model of B-Type sensor shows better performance for ethanol $\left(n_{a}=1.354\right)$ sensing. Conversely, X-Model of A-Type and V- Model of BType sensor for water $\left(\mathrm{n}_{\mathrm{a}}=1.33\right)$ sensing.

Previously, various kinds of PCF based structures are designed and analyzed with commercial software packages. Various 
numerical methods $[7,10,19,21]$ are employed. These numerical modelling methods are very popular in computational electromagnetism, where they provide accurate and reliable results. However, on experimental point of view development of proposed models still needs to be discussed. In our proposed structures, for high flexibility and low fabrication cost, same diameter air holes with conventional hexagonal arrangement are used in the cladding area. For the same reason, diameter of core holes is kept fixed. Smooth operability of the optical sensor depends on effective analyte (Water \& Ethanol) filling. The unique selective filling technique [22-23] permits infiltration of the air holes within PCF structure [24]. Also, previous experimental studies [2526] have demonstrated that cladding and core holes can be liquid filled. Therefore, fabrication of proposed structures is viable with the current advances in nano-fabrication techniques.

TABLE II

OVERALL PERFORMANCE OF PROPOSED SENSORS

\begin{tabular}{|c|c|c|c|c|}
\hline & & Biref. & Analyte & Sensing \\
\hline \multirow{6}{*}{ 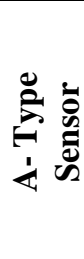 } & \multirow{2}{*}{$Z$ - Model } & \multirow{2}{*}{ High } & 1.33 & Low \\
\hline & & & 1.354 & High \\
\hline & \multirow{2}{*}{$X$ - Model } & \multirow{2}{*}{ High } & 1.33 & High \\
\hline & & & 1.354 & Medium \\
\hline & \multirow{2}{*}{$V-$ Model } & \multirow{2}{*}{ Low } & 1.33 & Medium \\
\hline & & & 1.354 & High \\
\hline \multirow{6}{*}{ 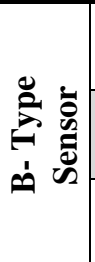 } & \multirow{2}{*}{ Z-Model } & \multirow{2}{*}{ Medium } & 1.33 & Medium \\
\hline & & & 1.354 & High \\
\hline & \multirow{2}{*}{ X-Model } & \multirow{2}{*}{ Medium } & 1.33 & Low \\
\hline & & & 1.354 & High \\
\hline & \multirow{2}{*}{$V$ - Model } & \multirow{2}{*}{ Low } & 1.33 & Medium \\
\hline & & & 1.354 & High \\
\hline
\end{tabular}

\section{CONCLUSION}

In this work, numerical analyses of dual analyte PCF based sensor models have been investigated with different core arrangements. This study has been proposed in favour of liquid sensing. Birefringence of proposed sensor types reaches up to $10^{-4}$ levels, where a sensitivity coefficient of ethanol varies between $32 \%-42 \%$. On the other hand, a sensitivity coefficient of water varies between $8 \%-25 \%$. Numerical results have shown that X-Model and Z-Model of A-Type sensor is an ideal structure for water and ethanol sensing, respectively. Thus, with such steady performance, the proposed dual analyte PCF structure can be employed for liquid sensing and other biological and chemical applications.

\section{REFERENCES}

[1] J. C. Knight, T. A. Birks, P. S. J. Russell, and D. M. Atkin, "All-silica single-mode optical fiber with photonic crystal cladding", Optics Letters, vol. 21, no.19,1996, pp. 1547-1549.

[2] P. St.J. Russell, "Recent Developments in Photonic Crystal Fibres", 2018 Conference on Lasers and Electro-Optics Pacific Rim (CLEO-PR), pp. Tu2E.1-2.
[3] X. Qi, S. Chen, Z. Li, T. Liu, Y. Ou, N. Wang, and Jing Hou, "Highpower visible-enhanced all-fiber supercontinuum generation in a sevencore photonic crystal fiber pumped at $1016 \mathrm{~nm}$ ", Optics Letters, vol. 43, no. 5, 2018, pp. 1019-1022.

[4] X. Feng, W. Feng, C. Tao, D. Deng, X. Qin, and R. Chen, "Hydrogen sulfide gas sensor based on graphene-coated tapered photonic crystal fiber interferometer", Sensors and Actuators B: Chemical, vol. 247, 2017, pp. 540-545.

[5] A. Yasli and H. Ademgil, "Geometrical comparison of photonic crystal fiber-based surface plasmon resonance sensors", Optical Engineering vol.57, no. 3, 2018, pp. 030801(1-10).

[6] R. Gao, D. F. Lu, J. Cheng, Y. Jiang, L. Jiang, J. D. Xu, and Z. M. Qi, "Fiber optofluidic biosensor for the label free detection of DNA hybridization and methylation based on an in-line tunable mode coupler", Biosensors \& Bioelectronics, vol. 86, 2016, 2016, pp. 321-329.

[7] H. Ademgil, "Highly sensitive octagonal photonic crystal fiber based sensor", Optik - International Journal for Light and Electron Optics, vol. 125, 2014, pp. 6274-6278.

[8] V.T. Hoang, B. Siwicki, M. Franczyk, G. Stepniewski, H.L. Van, V.C. Long, M. Klimczak, and R. Buczyński, "Broadband low-dispersion lownonlinearity photonic crystal fiber dedicated to near-infrared high-power femtosecond pulse delivery", Optical Fiber Technology, vol. 42, 2018, pp. 119-125.

[9] P. B. Kumar, K. Ahmed, D. Vigneswaran, F. Ahmed, S. Roy, D. Abbott, "Quasi photonic crystal fiber based spectroscopic chemical sensor in the terahertz spectrum: design and analysis", IEEE Sensors Journal, vol. 18, no. 24, 2018, pp. 9948 - 9954 .

[10] C. M. B. Cordeiro, M. A. R. Franco, G. Chesini, E. C. S. Barretto, R. Lwin, C. H. B. Cruz, and M. C. J. Large, "Microstructured-core optical fibre for evanescent sensing applications," Optics Express, vol. 14, no. 26, 2006, pp. 13056-13066.

[11] T. M. Monro, D. J. Richardson, and P. J. Bennett, "Developing holey fibres for evanescent field devices", Electronics Letters, vol. 35, no. 14, 1999, pp. 1188-1189.

[12] K. Naeem, Y. Chung and B. H. Kim, "Cascade Two- Core PCFs Based In-Line Fiber Interferometer for Simultaneous Measurement of Strain and Temperature", IEEE Sensors Journal, vol.19, no.9, 2019, pp.33223327.

[13] F. Q. Shi, Y. Y. Luo, D. R. Chen, J. J. Chen, Z. J. Ren and B. J. Peng, "A Dual- Parameter Sensor Based on the Asymmetry of Alcohol Filling the Photonic Crystal Fiber in Sagnac Loop Temperature", IEEE Sensors Journal, vol.18, no.15, 2018, pp.6188-6195.

[14] C. Zhou, H. K. Zhang, P. Song, J. Wang, C. G. Zhu, P. P. Wang and F. Peng, "Geometrically Structural Parameters Insensitive Fiber Sensor for Detection of Ethanol Concentration", IEEE Photonics Technology Letters, vol. 30, no. 23, 2018, pp. 2037-2039.

[15] M. Suganthya, B. K. Paul, K. Ahmed, Md. I. Islame, Md. A. Jabind, A. N. Bahar, M. S. M. Rajan, "Analysis of optical sensitivity of analytes in aqua solitions", Optik- International Journal for Light and Electron Optics, vol. 178, 2019, pp. 970-977.

[16] K. Ahmed, F. Ahmed, S. Roy, B. K. Paul, Mst. N. Aktar, D. Vigneswaran and Md. S. Islam, "Refractive Index- Based Blood Components Sensing in Terahertz Spectrum", IEEE Sensors Journal, vol.19, no.9, 2019, pp.3368-3375.

[17] Y. Yang, F. Yang, H. Wang, W. Yang and W. Jin, "TemperatureInsensitive Hydrogen Sensor with Polarization-Maintaining Photonic Crystal Fiber-Based Sagnac Interferometer", IEEE Journal of Lightwave Technology, vol. 33, no. 12, 2015, pp. 2566-2571.

[18] P. Hlubina, T. Martynkien, J. Olszewski, P. Mergo, M. Makara, K. Poturaj and W. Urbanczyk, "Spectral-domain measurements of birefringence and sensing characteristics of a side-hole microstructured fiber", Sensors, vol.13, no.9, 2013, pp.11424-11438.

[19] M. S. Islam, J. Sultana, K. Ahmed, M. R. Islam, A. Dinovitser, B. W. Him Ng, and D. Abbott, "A novel approach for spectroscopic chemical identification using photonic crystal fiber in the terahertz regime", IEEE Sensors Journal, vol. 18, no. 2, 2018, pp. 575-582.

[20] B. T. Kuhlmey, B. J. Eggleton, and D. K. C. Wu, "Fluid-filled solidcore photonic bandgap fibers", IEEE Journal of Lightwave Technology, vol. 27, no. 11, 2009, pp. 1617-1630.

[21] S. Padidar, V. Ahmadi, and M. Ebnali-Heidari, "Design of High Sensitive Pressure and Temperature Sensor Using Photonic Crystal Fiber for Downhole Application", IEEE Photonics Journal, vol. 4, no. 5, 2012, pp. 1590-1599. 
[22] F. Wang, W. Yuan, O. Hansen, and O. Bang, "Selective filling of photonic crystal fibers using focused ion beam milled microchannels", Optics Express, vol.19, no.18, 2011, pp.17585-17590.

[23] Y. Huang, Y. Xu, and A. Yariv, "Fabrication of functional microstructured optical fibers through a selective-filling technique", Applied Physics Letters, vol. 85, no. 22, 2004, pp. 5182-5184.

[24] S. Ertman, K. Rutkowska, and T. R. Wolinski, "Recent Progress in Liquid-Crystal Optical Fibers and Their Applications in Photonics", IEEE Journal of Lightwave Technology, vol. 37, no.11, 2019, pp. 25162526.

[25] M. Luo, Y. Liu, Z. Wang, T. Han, Z. Wu, J. Guo and W. Huang, "Twinresonance-coupling and high sensitivity sensing characteristics of a selectively fluid-filled microstructured optical fiber", Optics Express, vol. 21, no.25, 2013, pp. 30911-30917.

[26] R.M. Gerosa, D.H. Spadoti, C.J.S. de Matos, L.S. Menezes and M.A.R. Franco, "Efficient and short-range light coupling to index-matched liquid-filled hole in a solid-core photonic crystal fiber", Optics Express, vol.19, no.24, 2011, pp.24687-24698.

\section{BIOGRAPHIES}

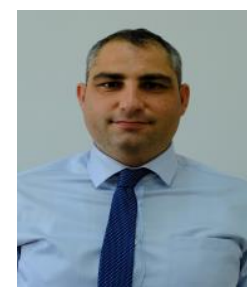

HUSEYIN ADEMGIL received his B.Sc. Degree in 2005 from Eastern Mediterranean University in Electrical and Electronic Engineering. He received the M.Sc. and $\mathrm{PhD}$ degree from the University of Kent, Canterbury, U.K., in 2006 and 2010, respectively. In 2009 July, he joined to the Kosova Telecommunication Company and assigned as a Project Manager. He has contributed in a number of projects such as FFTx and Planning and optimization of GSM Network. In September 2010, he joined European University of Lefke, where he is currently appointed as Head of the Computer Engineering Department. Dr. Ademgil was assigned to the TRNC Information and Communication Technologies Authority (BTHK) as a member of Board of Directors during 2012 May- 2019 May. He has been assigned on July 2019 to the Turkish Cypriot Electricity Authority (KIB-TEK) as a member of Board of Directors. He is a Senior Member of IEEE. 